\title{
Drawing the line: Drawing and construction strategies for simple and complex shapes in Williams syndrome and typical development
}

Kerry D. Hudson ${ }^{1}$ and Emily K. Farran ${ }^{2}$.

${ }^{1}$ School of Psychology and Clinical Language Sciences, University of Reading

${ }^{2}$ Department of Psychology and Human Development, Institute of Education

*Requests for reprints should be addressed to Kerry Hudson, Department of Psychology,

School of Psychology and Clinical Language Sciences, University of Reading, Harry Pitt Building, Earley Gate, Reading, Berkshire, RG6 6AL, UK, (e-mail: k.d.hudson @ reading.ac.uk). 


\begin{abstract}
In the typical population, a series of drawing strategies have been outlined, that progressively emerge during childhood. Individuals with Williams syndrome (WS), a rare genetic disorder, produce drawings that lack cohesion, yet drawing strategies in this group have hitherto not been investigated. In this study WS and TD groups drew and constructed (from pre-drawn lines and shapes) a series of intersecting and embedded figures. Participants with WS made use of the same strategies as the TD group for simple intersecting figures, though were less likely to use a typical strategy for more complex figures that contained many spatial relations. When replicating embedded shapes, the WS group used typical drawing strategies less frequently than the TD group, despite attempting to initiate a strategy that is observed in TD children. We conclude that individuals with WS show a particular difficulty with replicating figures that include multiple spatial relations. The impact of figure complexity and task demands on performance are discussed.
\end{abstract}




\section{Introduction}

Drawing strategies have been described as the "grammar of action" for drawing and refer to schemes for replicating figures using pre-determined rules and procedures (Ninio \& Lieblich, 1976, p. 846). In typical development, drawing ability improves throughout childhood. This has been attributed in part to a progression through a series of increasingly more mature drawing strategies (Feeney \& Stiles, 1996; Magnan, Baldy \& Chatillon, 1999). The drawings produced by individuals with Williams syndrome (WS), a rare genetic disorder, characteristically lack cohesion and contain errors resembling those made early in typical development (Bellugi, Lichtenberger, Jones, Lai \& St. George, 2000; Bertrand, Mervis \& Eisenberg, 1997; Georgopoulos, Georgopoulos, Kuz \& Landau, 2004). However, an understanding of the use of drawing strategies has not been formalised in WS and as such the strategies available to individuals with WS have not been related to the drawing strategies employed in typical development.

WS results from a hemizygous $1.6 \mathrm{Mb}$ microdeletion of approximately 26 contiguous genes on chromosome 7q11.23 (Nickerson et al., 1995; Tassabehji, 2003); with an estimated incidence of one in 7,500 (Strømme et al., 2002) to one in 20,000 (Morris et al., 1988) live births. The disorder is typified by mild to moderate learning difficulties (average IQ between 50 and 60), facial dysmorphology, hypersociability, cardiovascular dysfunction and an unusual cognitive profile that is characterised by a disparity between relatively strong linguistic ability and poor visuo-spatial ability (Ewart et al., 1993; Ferrero et al., 2007; Mervis \& John, 2008; Smoot, Zhang, Klaiman, Schultz \& Pober, 2005). It has been argued that linguistic ability is "spared" in WS (e.g. Bellugi Wang \& Jernigan, 1994) although this assumed that language develops from typical processes (Karmiloff-Smith, Brown, Grice \& Paterson, 2003). We now know that that linguistic ability develops at a faster rate than non- 
verbal ability (Jarrold, Baddeley \& Hewes, 1998), but that the development of some aspects of language is atypical (e.g. Thomas et al., 2001).

Poor visuo-spatial ability in WS was originally hypothesised to reflect a local-level processing preference (e.g. Bellugi, Sabo, \& Vaid, 1988) based on evidence from performance in drawing and construction tasks in which individuals with WS produced the local details without integrating these parts into the correct global, spatial arrangement. It is now recognised that a local level bias is not characteristic of all aspects of visuo-spatial ability; for example, performance on perceptual tasks such as the Children's Embedded Figures Test (Witkin et al., 1971), shows a typical balance of local and global processing in WS (Farran, Jarrold \& Gathercole, 2001, also see Deruelle, Rondan, Mancini \& Livet, 2006; Farran, Jarrold \& Gathercole, 2003; Pani, Mervis \& Robinson, 1999). The lack of global cohesion in visuo-spatial construction and drawing in WS, therefore, does not result from poor perception of the to-be-copied image, but likely reflects impairments in a number of underlying factors such as mental imagery and the comprehension of spatial relations, many of which are impaired in WS (e.g. Farran et al., 2001; Farran \& Jarrold, 2005). This study investigated the strategies employed to produce drawings and constructions in WS. In light of evidence that the incohesive drawings and constructions observed in WS resemble those seen in typically developing 4- and 5-year-old children (Bellugi et al., 1988; Bertrand et al., 1997), we sought to explore the underlying strategies that lead to poor drawing and construction in WS.

Drawing and construction involve the segmentation of the to-be-copied image into parts, followed by the integration of those parts to produce the image. Tada and Stiles (1996) explored drawing and construction in TD children aged 3;6 to 5 years and adults. Results demonstrated that systematically distinct strategies emerged during development and that a developmental transition lead to more mature strategy use as a function of age and figure 
complexity. Participants were presented with, in order of complexity, a plus, a cross and a three-line intersecting figure. These figures feature in the Test of Visual-Motor Integration (VMI, Beery, 1997) as an index of developmental level; age norms dictate reproduction of these figures is mastered at 4;1 years, $4 ; 11$ years and 5;9 years respectively. Tada and Stiles (1996) demonstrated that adults reproduced each figure using its constituent intersecting lines, e.g. centrally intersecting a horizontal and a vertical line to copy the plus figure (Unsegmented Part strategy). Two additional strategies were employed by the children. The least mature strategy, most often observed in children aged 3 to 4years, used shorter lines that radiated from a central point, i.e. the figure was segmented into the smallest possible independent units, that were then integrated into an holistic form (Central Point strategy). An intermediary strategy, most often observed in the 4 to 5 years olds, involved reproducing the figure using a mixture of unsegmented and segmented parts. For example, for a plus figure, a vertical line with two shorter lines originating from the centre-point were depicted (Mixed strategy). Strategy choice was dictated not only by age, but also by figure complexity, e.g. whilst the 3-4 year olds employed all three strategies to a similar extent for the plus figure, for the three-line intersecting figure, the majority of correct responses involved the Central Point strategy. In summary, whilst adults implement intersections, 3- to 5-year-old children often avoid drawing intersecting lines in favour of drawing junctions, but show some progression in their choice of junctions (Tada \& Stiles, 1996). The present study explores the use of these three strategies in individuals with WS.

According to the local processing hypothesis, individuals with WS fail to produce integrated drawings due to excessive attention to detail. In support of this, Bertrand et al. (1997) found that children with WS, when attempting to draw simple shapes and line combinations from the VMI, made integration errors that resembled those observed in 4- and 5-year-old TD children. Additionally, they showed that the ability to accurately integrate 
component parts of a figure was not fully available until 6 years in TD children. Although Bertrand et al. (1997) did not explore strategy use, if the ability to produce integrated drawings reflects strategy development, it is possible that individuals with WS employ strategies akin to those used by 4- and 5-year-old TD children. Alternatively, atypical development (such as the understanding of spatial relations) may impact the strategies employed to produce drawings and constructions in WS, such that they are qualitatively different from typical development. Georgopoulos et al. (2004) further examined the accuracy of figure copying using the VMI in children with WS and TD mental age (MA) matched controls aged 4; 1years ( $n=10$ in each group). Similar to Bertrand et al. (1997), the WS drawings resembled those of MA control group. Interestingly, the authors noted a group difference on a three-line intersecting figure only. For this figure, the individuals with WS adopted an adult-like Unsegmented Part strategy. This contrasted with the drawings of the MA-matched controls where the figure was segmented into small units, using less mature strategies (Central Point and Mixed strategies). As the Unsegmented Part strategy relies on an understanding of the gestalt of the figure (Feeney \& Stiles, 1996), this suggests that the local level bias observed in WS drawings does not originate from immature strategy use, at least for relatively simple, abstract figures. This raises the possibility that in some instances, a lack of coherence in WS drawings might be evident despite attempts to employ a mature drawing strategy. This would be indicative of qualitative differences in drawing ability in WS.

In contrast to the strategies employed for intersecting line figures, for embedded figures a single strategy emerges with development: the Centripetal Execution Principle (CEP; Magnan et al., 1999). The CEP dictates that for embedded figures (for an example, see Figure 2), copying commences from the external shape and continues progressively to the centre of the figure in order to replicate the model strategically. Each part of the figure is hierarchically replicated and the previous element anchors placement of the next part. The 
tendency to follow the CEP emerges at four years and increases in use, when from eight years old to adulthood the strategy is exclusively used (Bouziz \& Magnan, 2007; Magnan et al. 1999). Use of the CEP is fragile in children under eight years old when the standard procedure is modified, e.g. drawing subsequent to a delay, copying an embedded figure where some shapes have an increased line-weighting (therefore increased visual salience) or viewing non-CEP-like orders of superimposition of shapes (Bouziz \& Magnan, 2007; Magnan et al. 1999). In these instances children under eight years old will copy a figure that commences with the most salient element or will follow the order of superimposition that was viewed. In contrast, children over eight years of age can overcome these modifications to adhere to the CEP. Woody-Dorning and Miller (2001) have suggested that the ability to implement a strategy despite increased cognitive demand emerges with development, thus eight year olds can overcome attempts to disrupt the CEP despite the increased associated effort needed to do so. Siegler, Adolph and Lemaire (1996) argue that cognitive flexibility is important for use of strategies and that the most strategic behaviour results from possessing a range of strategies to solve the same problem, dependent upon task-demands. Strategic drawing of embedded figures using the CEP may be less frequent in individuals with WS as attentional control is poor in this group (Megnhini, Addona, Costanzo \& Vicari, 2010).

The clear developmental progression of strategy-use for replicating intersecting figures and embedded figures permits mapping of WS behaviour to TD levels of strategy-use so as to provide the first evidence of the availability and implementation of drawing strategies in WS. In this study, the influence of figure complexity on strategy-use was investigated for both drawing and construction in WS and TD groups. Experiment One (Drawing and Constructing Simple and Complex Intersecting Figures) focussed on strategies for producing intersecting figures. Experiment Two (Drawing and Constructing Complex Embedded Shapes) assessed strategy-use for embedded shape replication. 
Experiment One: Drawing and Constructing Simple and Complex Intersecting Figures

This task explored the manner that WS and TD participants parsed spatial arrays and reintegrated elements to form an holistic percept of the figure during drawing and construction. Participants were required to draw figures composed of intersecting lines (drawing condition) and to construct the figures using lines printed onto strips of acetate (construction condition). Recognisable intersecting figures can be drawn by four to five years (derived from the VMI) in TD children. The children employed in the current study were older than those employed by Tada and Stiles (1996). As such, although complexity is predicted to lead to a greater propensity to use less mature strategies in the TD group, this effect is predicted to be weaker than observed by Tada and Stiles (1996). The range of figure complexity has been increased in this study due to the inclusion of a four-line figure that was not used by Tada and Stiles (1996).

If the local bias observed in WS drawings (e.g. Bertrand et al., 1997) reflects the use of immature strategies, the WS group will show a high frequency of Central Point strategy use relative to the TD control group, as this involves segmenting figures into the smallest possible independent (local) units. However, Georgopoulos et al. (2004) reported some use of an Unsegmented Part strategy in WS. This appears to suggest that strategy use is not the source of the local-level bias in WS drawings, and thus predicts that a mixture of strategies will be employed by the WS group. Both strategy use and the ability to produce correct replications will be negatively affected by figure complexity for the WS and TD groups. Comparisons between the strategies used to support correct replications, as a function of 
complexity, will determine whether the WS group replicate figures in a typical or atypical manner.

When drawing, participants must plan the placement of lines and their relative length, whilst in construction the component elements are provided for the participant and errors in orientation or placement can be adjusted online. To date, although a local-level bias is reported in both drawing and construction tasks in WS (Farran et al., 2003), these two types of production ability have not been directly compared. If the manner that figures are viewed when drawn and constructed is homologous, then strategies will not differ between conditions in both groups. However, if the difficulty in drawing in WS is impacted by the graphomotor demands of planning each individual element, one might predict a higher preponderance of mature strategies and a weaker local processing preference in WS on the construction task, relative to the drawing task.

\section{Method}

\section{Participants}

Nineteen participants with WS were recruited from the Williams Syndrome Foundation UK. Diagnosis of WS in all participants had previously been confirmed by a clinician and a positive Fluorescence In Situ Hybridisation (FISH) test to ensure deletion of the elastin gene, observed in $95 \%$ of those with WS (de Souza, Moretti-Ferrereira \& Rugolo, 2007).

Nineteen TD non-verbal ability matched control participants were recruited from primary schools in Berkshire. Control participants were individually matched to the participants with WS using Raven's Coloured Progressive Matrices (RCPM; Raven, 1993). RCPM is a recognised measure of fluid intelligence, which has previously been used successfully as a matching measure when assessing visuo-spatial performance in developmental disorder groups such as autism and WS (e.g. Farran et al., 2003). The two 
groups did not differ in RCPM scores, suggesting that matching was adequate, $t(36)=.37$, $p=.71$.

Both groups completed the British Picture Vocabulary Scale II (BPVS II; Dunn, Dunn, Whetton \& Burley, 1997) that assessed verbal ability (receptive vocabulary). This allowed for a fuller picture of each participant's cognitive ability to be gained and underlined the disparity of linguistic and spatial ability in WS. Table 1 illustrates chronological age (CA), BPVS and RCPM scores for both groups.

\section{$<$ Table 1 about here $>$}

\section{Apparatus and Materials}

All figures could be completed using long $(7.6 \mathrm{~cm})$ and/or short lines $(3.8 \mathrm{~cm}$; half the length of a long line). There were four experimental figures in total that became progressively more complex due to the orientation of the component lines and the number of intersections. Additional control figures (the Lateral ' $\mathrm{T}$ ' and the Slanted ' $\mathrm{T}$ ') were included to examine whether participants were able to replicate junctions (the core ability necessary for adopting a Central point strategy) and used both long and short lines to assess appreciation of relative line-lengths. The experimental and control figures can be seen in Figure 1.

\section{<Figure 1 about here>}

\section{Design and Procedure}

Experiments One and Two were run concurrently. Administration of the BPVS and RCPM was interleaved between Experiments One and Two, with order of both the standardised tests and Experiments counterbalanced across participants. In Experiment One participants were first shown the six target figures (four experimental and two control) in a randomised order for the drawing condition. A fixed order of conditions was used, drawing 
was completed first to avoid priming strategies from the construction condition that draws attention to the distinct nature of the component parts. In both conditions the model figure was presented centrally on a separate A4 sheet of paper in a portrait orientation and remained in view throughout the trial. When drawing, participants were asked to replicate the model exactly on A4 paper, using a pencil. Errors could be corrected by participants if they were recognised, analysis was conducted on the final solution that participants offered. The order and orientation of each line that was drawn and the strategy used was recorded for each figure. In the construction condition lines were presented in front of participants in groups according to the size of lines, the side of grouping of line-type (left or right) was counterbalanced between participants. Participants were given four long lines and eight short lines with a $0.5 \mathrm{~cm}$ surrounding transparent border. This was the maximum number of lines needed to create the most complex figure (four-line figure) using any of the possible strategies. In the pre-trial familiarisation phase participants watched as the experimenter manipulated two long and two short lines into a single rectangular form. This shape did not resemble any of the models but illustrated how the line-types could be manipulated to copy a figure. Participants were then told that instead of drawing the figures that they were to construct them from any of the types of lines necessary to replicate the target exactly. The six figures were presented in a randomised order; subsequent to construction of each figure the experimenter regrouped the lines that were used, by line-type. The types of lines that were used, the order of placement of each part and the strategy used were recorded

\section{Results}

Reproductions were deemed "correct" if there was evidence of appreciation of relative line lengths and replications were recognisable in terms of configuration. Failure to achieve this led to an Incomplete (Incorrect) classification. In the construction condition, 
participants were not penalised for using incorrect line-types if relative line-lengths resembled the model, for example, two short lines could be used in place of two long lines when intersecting. This was due to the potential for stricter coding of constructions relative to drawings that would artificially lead to poorer construction scores as an artefact of stringent coding of lines used, whereas drawings were coded on the basis of configuration.

\section{Analysis of Control Figures}

Control figures assessed whether participants could form junctions. This ability is necessary in order to produce integrated figures using the most basic (Central Point and Mixed) strategies. All participants correctly drew the Lateral-T and when drawing the Slanted-T all but one TD participant correctly replicated the figure (this participant subsequently constructed all experimental figures using the Unsegmented Part strategy). When constructing the Lateral-T, six participants with WS and three TD participants incorrectly constructed this figure. Similarly, when constructing the Slanted-T, four participants with WS and one TD participant failed to accurately replicate this figure. However, all of these participants subsequently showed evidence of junction use when replicating the experimental stimuli. Both groups therefore possessed the core ability of junction-formation necessary to replicate the experimental figures at the least developmentally advanced levels (Central Point- and Mixed).

\section{Analysis of Experimental Figures}

All correctly completed figures employed recognisable strategies, i.e., no participants used a strategy other than those described by Tada and Stiles (1996). There was no evidence of a lack of integration in any replication in the WS or TD groups. Incorrect replications were 
due to replications produced with additional parts or too few parts. These figures were not classified in terms of strategy use, and so received a classification of Incomplete.

\section{Accuracy analysis}

Accuracy was assessed in terms of number of correct replications, irrespective of the strategy employed. A ceiling effect was observed in the TD group in the drawing condition where all participants achieved the maximum score of four correct replications. In the remaining conditions both groups performed significantly differently from floor and ceiling ( $p<.05$ for all). A group (WS, TD) by condition (drawing, construction) ANOVA was conducted on the number of correct replications (out of four). There was a significant effect of group $F(1,36)=11.28, p=.002, \eta_{p}{ }^{2}=.24$ with the TD group $(M=3.74, S E=.13)$ achieving a higher score compared to the WS group $(M=3.13, S E=.13)$, although this difference is less than one point. There was a significant effect of condition $F(1,36)=8.79, p=.005, \eta_{p}{ }^{2}=.20$; drawing $(M=3.66, S E=.09)$ led to greater accuracy than construction $(M=3.21, S E=.14)$. There was no group by condition interaction, $F(1,36)=.27, p=.60$. This was due to comparable accuracy in both groups in both condition (WS: Drawing: $M=3.32, S E=.13$, Construction: $M=2.95, S E=.19$; TD: Drawing: $M=4.00, S E=.13$, Construction: $M=3.47$, $S E=.19)$.

\section{Strategy-type analysis}

The accuracy analysis above showed that the WS group accurately completed fewer figures than the TD group. Taking a more analytic approach, the following analysis compares the frequency of use of each strategy type for drawing and construction across groups (for correctly replicated figures only). Table 2 illustrates participants' frequency of use of each strategy-type (note that Incomplete responses are displayed in Table 2, but were not entered into the analyses). Data were entered into Chi Square and Fisher's Exact analyses. The relationship between strategy-type (three strategy types: Unsegmented Part, Mixed, Central 
point) and group (two groups: WS, TD) was analysed separately for each figure in each condition, drawing and construction (plus and cross figures in the drawing conditions were not analysed due to consistent use of the Unsegmented Part strategy by all WS and all but one TD participants). This showed that the strategy choices for each figure in drawing or construction conditions was not related to group membership ( $p>.05$ for all). Therefore, when individuals with WS correctly replicated a figure, the choice of strategy was not different from those employed by the TD group for each figure.

$<$ Table 2 about here $>$

\section{Figure Complexity analysis}

Analysis of strategy-types demonstrated that although individuals with WS produced fewer correct replications than the TD group, the strategies used to produce correct replications showed similar patterns across groups. The following analyses introduce figure complexity as a factor. Complexity increased in the figures due to the number of spatial relations and component lines. This might have not only affected participants' strategy choice, but also their ability to successfully reproduce the figure (i.e. accuracy), each of which are addressed in turn. First, chi-square and Fisher's Exact analyses were conducted to explore the relationship between figure complexity (plus, cross, three-line and four-line figure) and accuracy (correct or incomplete). Separate analyses were conducted for each group, in each condition. When drawing, the WS group showed a significant relationship between accuracy and figure complexity, $\chi^{2}(3)=20.32, p<.001$. Correct reproductions were more frequently produced than incomplete reproductions when drawing the plus and cross, the opposite pattern was observed for the four-line figure. Complexity also affected accuracy in constructions in the WS group, $\chi^{2}(3)=14.66, p=.002$. This was due to frequent correct constructions of the plus and frequent incomplete replications of the four-line figure. The TD 
group principally produced correct reproductions in drawing (no incorrect drawings were produced) and construction $\left(\chi^{2}(3)=5.78, p=.12\right)$, accuracy was unaffected by complexity in the TD group.

Second, chi-square analyses were conducted to explore the relationship between figure complexity (plus, cross, three-line, four-line) and strategy-type (Unsegmented, Mixed, Central Point) (Incomplete figures were not included in this analysis). Separate analyses were conducted for each group in each condition. In line with the association between complexity and accuracy above, when drawing the WS group's use of strategies also depended upon the complexity of the model, $\chi^{2}(6)=20.96, p=.002$. Four-line figures led to less use of Unsegmented Part, Mixed and Central Point strategies whereas plus and cross figures led to a greater use of an Unsegmented Part strategy and less use of the Mixed strategy. However, contrary to the association between complexity and accuracy above, complexity of models did not affect strategy-use when constructing figures in the WS group, $\chi^{2}(6)=3.57, p=.73$ ). Although complexity did not affect accuracy in the TD group, it did affect strategy use for both drawing $\left(\chi^{2}(6)=14.56, p=.02\right)$ and construction conditions $\left(\chi^{2}(6)=23.06, p=.001\right)$. When drawing the plus and cross figures an Unsegmented Part strategy was used. The three-line figure led to less use of an Unsegmented Part strategy and also a greater instance of a Mixed strategy. When constructing figures, three-line figures led to less use of the Unsegmented Part strategy and an increased use of a Central Point strategy, the use of the Mixed strategy also increased in the four-line figure.

\section{Discussion}

The results show that overall accuracy scores were higher in the TD group than the WS group. This was because figure complexity impacted both accuracy and strategy choice in the WS group, but impacted strategy choice only in the TD group. The group difference 
occurred despite ceiling effects in the TD group in the drawing condition, and so for this condition it is possible that group differences were attenuated. Analysis of the frequency of strategies that were used revealed that, when figures were correctly reproduced, individuals with WS displayed the same pattern of strategies as the TD group. Both groups frequently used an adult-like Unsegmented Part strategy when drawing and constructing figures. This is the first time that such a result has been shown and suggests that although drawing ability is poor in WS (Bellugi et al, 2000), typical drawing strategies are available and can be appropriately used to replicate intersecting figures. It was hypothesised that, if the local bias in WS drawings reflects immature strategy use, the WS group would use less mature strategies, i.e. a Central Point or Mixed strategy. The data do not support this hypothesis and suggests that the local bias observed in drawing in WS is not the result of immature strategy use. Incomplete reproductions were not a result of disorganised configurations. This appears to counter previous findings in WS research of a local processing bias on drawing and construction tasks (e.g. Bertrand, Mervis \& Eisenberg, 1997; Farran et al., 2001). The series of figures employed in the current task became increasingly complex on account of the orientation and number of component lines of each figure. Complexity impacted strategy use for both groups, i.e. both groups began to introduce less mature strategies as a function of increased complexity in both drawing and constructing conditions. However, complexity also affected the ability to produce accurate solutions in the WS group, but not the TD group. Specifically, whilst the majority of the TD group produced accurate figures throughout, the WS group found the four-line figures difficult to draw and construct. Thus, it seems that complexity could be overcome by the TD group through a change in strategy and although this method was also employed by the WS group, it did not suffice for the most complex figure, resulting in a high frequency of incomplete replications. This suggests that beyond a certain level of stimulus complexity, the WS group may fail to parse the arrangement of parts 
of the figure in order to produce a replication, suggesting a difficulty with copying multiple spatial relations. This raises the possibility that the 'threshold' for adjusting to stimulus complexity in WS is unusually low, relative to typical development. This might also explain inconsistencies between this study and those that report integration errors in WS, as many such studies (although not all) used more complex figures than those employed here.

Tada and Stiles (1996) showed that the strategies were homologous between drawing and construction, but in the current study accuracy was higher when drawing than constructing. If differences between drawing and construction accuracy are a product of increased complexity, the effect observed in our study might reflect the inclusion of a fourline figure, which was not included by Tada and Stiles (1996). However, this argument is not supported by both groups; the WS group, but not the TD group, showed an association between accuracy and complexity. It appears then that this effect was driven by the WS group and although this is not supported statistically (the effect of condition did not interact with group), exploration of group means of accuracy suggest that this might be due to a lack of power. Errors in construction may have resulted from the more multi-phasic nature of constructing figures from pre-drawn lines. That is, greater checking between the available lines, those selected for the copy and the line-types in the model figure is needed in order to choose the correct elements and place these with the proper spatial relations.

\section{Experiment Two: Drawing and Constructing Complex Embedded Shapes}

The previous experiment suggested that individuals with WS failed to produce accurate replications when figures contained increasingly complex spatial relations whereas the TD group overcame changes in complexity by implementing a less mature strategy. Experiment Two investigated complexity in terms of relations between shapes rather than 
relations between lines (as in Experiment One) and examined use of the Centripetal Execution Principle (CEP; Magnan et al., 1999). As stated earlier, for embedded figures, strategic replication involves the CEP, in which participants commence copying from the external shape and continue progressively to the centre of the figure in order to replicate the model. The emergence of use of the CEP is unknown in WS.

This experiment also addressed methodological issues with previous experiments that have examined the CEP in the typical population. Such studies failed to maintain shape-types between figures, for example different exemplars of triangles were used which could have affected participants' perception and recognition of the figures (Satlow \& Newcombe, 1998). Previous studies also presented figures with only diamond and triangle shapes as the external shape of figures. However, because successful drawing of shapes containing oblique lines occurs later in development than the ability to draw curvilinear and horizontal or vertical lines (Piaget, 1948), this may have lead to an inability to commence externally when drawing embedded figures, giving the appearance of a failure to use the CEP. As such, in this Experiment, we have ensured constancy of shapes between figures and have systematically counterbalanced the relative position of graphically-demanding shapes (such as diamonds or triangles due to the inclusion of oblique lines) within the embedded figure.

The CEP is not reliably employed until children are eight years old (Bouaziz \& Magnan, 2007), therefore emerging relatively later in typical development than the strategies assessed in Experiment One. One could argue that the ability to replicate embedded figures is relatively more developmentally-advanced and thus a more complex ability than replication of intersecting figures (as in Experiment One). As such, on account of reports of poor drawing ability in WS (e.g. Bertrand et al., 1997) and a lower threshold for complexity (Experiment One), it was hypothesised that the WS group would use the CEP less frequently than the TD group when drawing and constructing. Furthermore, as the CEP involves 
hierarchical processing of the relationships between the parts, it is likely that without this strategy the WS group will show evidence of a part-based or local processing approach. In addition, the CEP may not be used exclusively by the TD group as participants are younger than eight years, when the CEP is universally utilised (Bouaziz \& Magnan, 2007).

Construction was hypothesised to lead to greater CEP-use in the WS group due to the reduced graphomotor demands of the task. Furthermore, as participants are provided with the individual shapes as segmented parts, this facilitates accurate segmentation of the model, thus providing an advantage over the drawing condition Alternatively, both groups may use the CEP less frequently when constructing figures due to visual correctness of the construction being achieved regardless of the order of superimposition; any order of superimposition results in a figure that resembles the model.

Method

\section{Participants}

Participants from Experiment One also completed this task, in the same testing session.

\section{Apparatus and Materials}

For each condition (drawing and construction) the same set of four figures were presented centrally on A4 sheets of paper in a portrait orientation each comprising of four embedded shapes; a square, diamond, circle and triangle. Each figure was $12 \mathrm{~cm}$ in height with an internal area of $144 \mathrm{~cm}^{2}$ (Figure 2). The individual shape appeared in each position from the centre once, this meant that certain shapes could not be adjoined as the forms became indistinct. A triangle, for example, could not be encompassed by a square as the base of the triangle was occluded by the square, which could have lead participants to fail to appreciate the distinct nature of the shapes. This could have been remedied by adjusting the form of the shape, such as using a scalene in place of an equilateral triangle, though the forms 
would not be constant between each figure which is potentially confounding. Additionally, four control figures were drawn and constructed that contained only two shapes to ensure that participants could create embedded figures. In the construction condition, each component shape was presented on separate A4 sheets of acetate. These could be overlain to recreate the embedded figure.

\section{$<$ Figure 2 about here>}

\section{Procedure}

Participants completed the control condition first to ensure that simple figures could accurately be embedded under both drawing and construction conditions. The construction condition was presented after the drawing condition in both control and experimental tasks as Magnan, et al. (1999) indicated that the order of superimposition of shapes in a construction condition could prime strategy use when drawing. For both conditions participants were shown the target figure, which remained in view throughout replication. No prompts were given as to the nature of the figure so as not to inform local or global processing styles (for example participants were not told that the figure was a larger shape made of smaller shapes or vice versa). When drawing, participants were instructed to copy the figures exactly onto a sheet of A4 paper using a pencil. Self-correction of errors was permitted and participants' final solutions were used for analysis. The drawing quality of copies was not assessed, instead it was noted whether the CEP was adhered to (the order to shape replication), namely when it was clear that participants were sequentially replicating component shapes from the exterior to the interior shape.

In the construction condition participants were presented with the shapes that comprised the model, printed onto separate acetate sheets and scattered randomly in front of them. Participants were told that instead of drawing the figure they were to construct it from the parts to copy the model. Participants constructed the figures, presented in a random order, 
by placing the acetate sheets in a box (to stabilise the acetate sheets). Perceived errors could be corrected by re-ordering the transparencies within the box, only final solutions were analysed. The order in which each of the shape elements were placed was recorded for each completed model indicating adherence to the CEP.

\section{Results}

Analysis of Control Figures

A total score out of four was awarded for CEP-implementation (in this instance, commencing from the external shape of the model and then replicating the central shape) in each condition. One point was awarded for successful CEP-use per figure. The chance level of implementing the CEP for these figures was 50\% (a score of two out of four). In both conditions, both groups differed significantly from chance, $p<.05$. A group (WS, TD) by condition (drawing, construction) ANOVA was performed on CEP-use scores out of four. There were no main effects of group $(F(1,36)=.27, p=.61)$, condition $(F(1,36)=.64, p=.43)$ or an interaction of group and condition $(F(1,36)=.04, p=.84)$. When drawing, the WS group used the CEP an average of 2.89 times ( $S E=.33$ ) with the TD grouping using the strategy an average of 3.11 time $(S E=.33$ ). When constructing figures the WS group used the CEP 3.16 times $(S E=.22)$ and the TD group used the strategy in $3.26(S E=.22)$ instances out of a maximum of four. This suggests that both groups were able to form embedded figures and adhere to the CEP for simple control figures. Therefore, any differences in the more complex figures were unlikely to be due to the inability to hierarchically place elements of a model in drawing and construction.

\section{Analysis of CEP-Use in Experimental Figures}

A total score out of four was awarded for implementation of the CEP, as in analysis of the control figures. The chance level of implementing the CEP for each figure was 1:24 (there are 24 possible orderings of four shapes, only one of which adheres to the CEP). In both 
conditions, both groups showed performance significantly different from floor, ceiling and chance $(p<.05)$. A group (WS, TD) by condition (drawing, construction) ANOVA was conducted on CEP-use scores (out of four). There was a significant effect of group, $F(1,36)=8.36, p=.01, \eta_{p}{ }^{2}=.19$, with the TD group $(M=2.87, S E=.33)$ implementing the CEP significantly more than the WS group $(M=1.53, S E=.33)$. There was no main effect of condition $(F(1,36)=.37, p=.55)$ or interaction of group by condition $(F(1,36)=.2 .51, p=.12)$.

\section{Analysis of Failure to Implement the CEP}

Analysis was conducted on instances in which the CEP was not used. This determined whether the strategy was initiated but not completed or whether no attempt was made to implement the CEP. The starting location of replication when the CEP was not used was classified as External (outermost shape), Internal (innermost shape) or Other (a shape that was neither internal nor external). For each participant, a percentage of each starting location was then calculated for all replications in which the CEP was not used (see Figure 3). A group (WS, TD) by condition (drawing, construction) by location (internal, external, other) ANOVA was conducted on the percentage of participants' starting locations. There was no effect of group $(F(1,6)=3.00, p=.13)$ or condition $(F(1,6)=3.00, p=.13)$. There was a significant effect of location, $F(2,12)=8.07, p=.006, \eta_{p}{ }^{2}=.57$ because replication began significantly less with External $(M=26.05 \%, S E=4.01 \%)$ or Internal $(M=27.10 \%, S E=1.98 \%)$ shapes than Other shapes $(M=46.28 \%, S E=3.49 \%)$. The main effect of location interacted with group, $F(2,12)=9.82, p=.003, \eta_{p}{ }^{2}=.62$ due to a significant group difference between External and Internal shape-use, but not Other shape use. The WS group $(M=38.91 \%, S D=$ $14.55 \%$ ) commenced with the External shape significantly more than the TD group $(M=13.19 \%, S D=6.73 \%), F(1,6)=10.30, p=.02$. The TD group $(M=58.54 \%, S D=4.47 \%)$ commenced with an Internal shape significantly more than the WS group $(M=34.02 \%, S D=$ 
$13.24 \%), F(1,6)=12.31, p=.01$. There was a significant interaction of location by condition, $F(2,12)=12.72, p=.001, \eta_{p}{ }^{2}=$. . This was the result of significantly greater use of the External shape in drawing $(M=38.80 \%, S D=19.64 \%)$ compared to construction $(M=13.30 \%, S D=$ $16.59 \%), t(7)=6.44, p<.001$, but no effect of condition for Other and Internal shape use. There was a significant interaction of group by condition by location $F(2,12)=8.71, p=.005, \eta_{p}{ }^{2}=.59$ (Figure 3). The TD group began drawing with the Internal shape $(t(6)=2.65, p=.04)$ and constructions commenced with Other shapes $(t(6)=6.00, p=.001)$ significantly more than the WS group. The WS group started drawings with External $(t(6)=4.07, p=.007)$ and Internal $(t(6)=2.68, p=.04)$ shapes significantly more than the TD group. The interaction of condition by group $F(1,6)=3.00, p=.13$ was not significant.

\section{$<$ Figure 3 about here>}

\section{Discussion}

TD individuals used the CEP significantly more than the WS group; this reiterated the effect of complexity seen in Experiment One. Less strategy-driven copying is therefore seen in individuals with WS when figures are relatively complex due to spatial relations between both shapes and lines. The failure of the WS group to implement the CEP as frequently as the TD group was unlikely to be related to the inability to place elements hierarchically; CEP-use was not different between groups when figures comprised of two shapes. Individuals with WS therefore possess the ability to embed figures but do not use the CEP frequently when embedding multiple figures.

When individuals with WS did not draw using the CEP, the strategy was initiated but not completed. Participants with WS were most likely to start with the external shape and fail to progress centripetally whereas the TD group commenced with an internal shape. Therefore the WS group attempted to use the CEP but failed to successfully implement the strategy 
whereas the TD group, when errors were made, did not attempt the CEP. This interestingly suggests that group differences emerged in the process of replicating four-shape figures that were absent in the control figures. This suggests, as in Experiment One, that there is a threshold in the ability to overcome figure complexity to successfully replicate a figure in WS, which appears to be lower than that observed in TD controls of the same level of nonverbal ability. This group difference may be related to attentional control when using the CEP, poor planning of placement of parts or may be a manifestation of perceptual atypicalities resulting from understanding of multiple spatial relations (such as crowding). This result is also surprising as the external shape of a figure represents a global-level feature. If the local processing preference was evidenced, this shape would not have been drawn initially by individuals with WS. A local processing preference would predict performance in the WS group to resemble copying in the TD group, where drawing commenced from internal elements when the CEP was not used. This suggests that even when the CEP is not used in WS, the CEP is initiated and fails to evidence a local-level preference despite the visuo-constructive demands of the task.

When comparing CEP-use between drawing and construction, both groups used the CEP equally frequently in both conditions. This suggests that the CEP is available under differing task demands to replicate embedded figures. Use of the CEP when constructing highlights the pervasive nature of the strategy, as any order of superimposition of shapes would result in a visually correct copy of the model. The use of the CEP in this instance reveals the adherence of the CEP when the strategy need not be used to accurately replicate the model. In support of Bouaziz and Magnan (2007), the CEP is a stable strategy and the outward to innermost progression of copying can be used when drawing and constructing embedded figures. This is the first time that use of this strategy has been reported in an 
atypically developing group, although further research is required to determine why the CEP was initiated but not always completed when drawing.

\section{General Discussion}

The current studies provided the first evidence of drawing and construction strategyuse in WS by examining the production of intersecting line figures (Experiment One) and embedded figures (Experiment Two). Results showed that individuals with WS, although less accurate than their TD peers, use typical strategies to produce strategies inline with nonverbal ability-level (derived from RCPM), but only when figures do not contain numerous spatial relations between parts and therefore are not complex. To explain, when drawing and constructing figures containing two embedded shapes, individuals with WS were able to use the CEP to the same extent as TD individuals. Further to this, individuals with WS used the same types of strategies as the TD group when drawing and constructing two- and three-line intersecting figures. In contrast, performance in the WS group became divergent from the TD group when drawing and constructing figures containing four intersecting lines and when drawing four-shape embedded figures; in these instances the WS group used a known strategy less frequently than the TD group. Although individuals with WS possess the ability to use strategies, increases in figure complexity have a stronger effect on performance than their typically developing peers, suggestive of different thresholds at which strategies are no longer effective in supporting accurate figure replication. This effect of figure complexity is not necessary related to the graphic demands of the component lines of the figures. Individuals with WS have a difficulty coding oblique orientations, therefore replication of figures containing these lines would be expected to be poor (Palomares, Landau \& Egeth, 2009). The cross, therefore, should be more demanding to replicate due to the inclusion of oblique lines that increase graphic planning demands relative to horizontal or vertical lines 
(Chen \& Levi, 1996). Tada and Stiles (1996) showed that the number of recognisable replications (in terms of accuracy, segmentation and configuration) decreased when a plus figure was orientated at $45^{\circ}$ in 3-5 year olds, the current data fails to support this in a WS group and with six year old TD children. This was not observed in this study in terms of strategy-use, as both groups employed a mature, Unsegmented Part strategy. This suggests that intersections can still be recognised when presented in oblique orientations, despite the relative difficulty of perception of this orientation in WS and TD groups (Gentaz et al., 2001). Tada and Stiles (1996) reported that the three-line figures educed a range of strategies in TD groups, with a lesser instance of Unsegmented Part replications. Conversely, Georgopoulos et al. (2004) reported Unsegmented Part-like strategies for copying this figure in a WS group and Central Point-like strategies in TD children. The current data supports this finding in a larger WS group, although Unsegmented Part strategies predominated in all figures produced by the TD group, suggesting that schema for drawing are more advanced at a younger age than previously reported. The advanced performance of the TD group may be the result of preferential implementation of implicit graphic schema for replicating well-rehearsed figures such as cross-like figures that children are familiar with through schooling as ceiling effects were observed when considering the frequency of strategy-use (Karmiloff-Smith, 1990; Phillips, Hobbs \& Pratt, 1978). Less familiarity with complex models may have resulted in the use of less developmentally advanced strategies. The TD group may have adapted schema that already existed to extend these to complex figures in both drawing and construction.

Further to less frequent strategy-use when figures contained many spatial relations between lines, individuals with WS were also less strategy-driven when drawing embedded figures where complexity resulted from relations between shapes. However, when the CEP was not used, during drawing the WS group more frequently commenced with the external shape but failed to progress centripetally, compared to the TD group. This is an important 
finding and warrants further research to determine why the strategy was not maintained. Failure to proceed with the CEP may reflect a form of crowding during drawing in which the external element is readily identified but internal elements become indistinct and confused (see Levi, 2008 for a review), control figures may not have contained enough elements induce a crowding effect. Construction led to comparable CEP-use between groups, despite visual correctness of constructions without use of the CEP. Bouaziz and Magnan (2007) reported greater CEP-use when drawing compared to construction only when the lineweighting of the component-shapes was manipulated in an attempt to disrupt CEP-use, i.e. when attention was drawn to the visual salience of the weighted-line shapes. The CEP may be discovered through trial and error, to be the least effortful means of replicating embedded figures (Crowley, Shrager \& Siegler, 1997). Less frequent CEP-use in the WS group relative to the TD group, may result from a utilisation deficiency (Miller, 1990) in which attempts to implement the CEP hinders the ability to strategically draw. Cognitive flexibility permits the most strategic behaviour to result from possessing a range of strategies to solve the same problem, dependent upon task-demands (Siegler, 1987; Siegler et al., 1996). As such, the CEP may be discovered through trial and error, to be the least effortful means of replicating embedded figures (Crowley, Shrager \& Siegler, 1997). However, participants with WS appear not to have reached this level of recognition, though can commence drawing with the external element. Planning the strategy to replicate figures can be slow and serial in nature, it is also demanding of working memory and attention which is poor in WS (Crowley et al., 1997; Menghini et al., 2010). The WS group have therefore not recognised the CEP as the most parsimonious means of drawing embedded figures.

Both experiments suggest that individuals with WS are less strategy-driven when copying figures with many spatial relations between parts (lines and shapes). Toomela (2002) suggested that drawing ability related to block construction and mental rotation ability in 2- 
11 year old TD children. Performance on these tasks is poor in WS, particularly when stimuli are complex (Hoffman et al., 2003; Stinton, Farran \& Courbois, 2008), this may also be due to the need to understand multiple spatial relations. Bertone, Mottron, Jelenic and Faubert (2005) suggested that in autism the complexity of stimuli (static and dynamic orientation gratings) affects feature-integration, reflecting atypical neurointegration in the visual cortex that is unrelated to local- or global-level processing. The effect of figure-complexity in WS may also have neurobiological underpinnings that warrant further research. If featureintegration is also poor in WS then selection and execution of a graphic plan may be poor. This is supported by evidence that errors in children's drawings are largely the result of planning difficulties (Broderick \& Laszlo, 1987; 1988). It may be that construction permits exploration of strategies more than drawing as graphic plans can be updated online (as parts can be moved in construction), leading to more frequent strategy-use (Crowley et al., 1997).

In summary, this study provides the first evidence of graphic strategy-use in WS and extends the literature on strategy use in typical development. TD children showed use of more developmentally advanced strategies when drawing and constructing embedded and intersecting figures than previous literature suggested. Individuals with WS showed reduced accuracy, but were able to use the same types of strategies as the TD group. This is an important finding that argues against a local processing preference and instead performance in WS may be better explained by a failure to reproduce multiple spatial relations, or a reduced tolerance to figure complexity. Further research is needed to determine whether this is the result of poor graphic planning in WS. Planning dysfunction in WS may also explain less frequent CEP-use when replicating embedded figures than the TD group as the strategy can be initiated, but not always completed. The current studies suggest that poor drawing 
performance in WS is best explained by a failure to replicate multiple spatial relations, though more research is needed to investigate this. 


\section{References}

Beery, K.E. (1997). The Beery-Buktenica Developmental Test of Visual Motor-Integration (VMI).New Jersey, USA: Modern Curriculum Press.

Bellugi, U., Lichtenberger, L., Jones, W., Lai, Z., \& St. George, M. (2000). The neurocognitive profile of Williams Syndrome: A complex pattern of strengths and weaknesses. Journal of Cognitive Neuroscience, (12, Supplement), 7-29.

Bellugi, U., Sabo, H., \& Vaid, J. (1988). Spatial deficits in children with Williams syndrome. In J. Stiles-Davis, U. Kritchevshy, \& U. Bellugi (Eds.), Spatial Cognition: Brain Bases and Development (pp. 273-297). Hillsdale, New Jersey: Lawrence Erlbaum.

Bellugi, U., Wang, P.P., \& Jernigan, T.L. (1994). Williams Syndrome: An unusual neuropsychological profile. In S.G. Broman, J. (Ed.), Atypical Cognitive Deficits in Developmental Disorders: Implications for Brain Function. Hillsdale, NJ: Lawrence Erlbaum Associates.

Bertone, A., Mottron, L., Jelenic, P., \& Faubert, J. (2005). Enhanced and diminished visuospatial information processing in autism depends on stimulus complexity. Brain, $128(10), 2430-2441$.

Bertrand, J., Mervis, C.B., \& Eisenberg, J.D. (1997). Drawing by children with Williams syndrome: A developmental perspective. Developmental Neuropsychology, 13, 4167.

Bouaziz, S., \& Magnan, A. (2007). Contribution of the visual perception and graphic production systems to the copying of complex geometrical drawings: A developmental study Cognitive Development, 22(1), 5-15.

Broderick, P., \& Laszlo, J.I. (1987). The drawing of squares and diamonds: A perceptualmotor task analysis. Journal of Experimental Child Psychology, 43, 44-61. 
Broderick, P., \& Laszlo, J.I. (1988). The effects of varying planning demands on drawing components of squares and diamonds. Journal of Experimental Child Psychology, $45(1), 18-27$.

Chen, S., \& Levi, D.M. (1996). Angle judgment: Is the whole the sum of its parts? Vision Research, 36(12), 1721-1735.

Crowley, K., Shrager, J., \& Siegler, R.S. (1997). Strategy discovery as a competitive negotiation between metacognitive and associative mechanisms. Developmental Review, 17, 462-489.

Deruelle, C., Rondan, C., Mancini, J., \& Livet, M.-O. (2006). Do children with Williams syndrome fail to process visual configural information? Research in Developmental Disabilities, 27, 243-253.

Dunn, L.M., Dunn, L.M., Whetton, C., \& Burley, J. (1997). The British Picture Vocabulary Scale 2nd edition. Windsor, U.K.: National Foundation for Educational Research.

Ewart, A.K., Morris, C.A., Ensing, G.J., Loker, J., Moore, C., Leppert, M., \& Keating, M. (1993). A human vascular disorder, supravalvular aortic stenosis, maps to chromosome 7. Proceedings of the National Academy of Sciences of the United States of America, 90(5), 3226-3230.

Farran, E.K. (2005). Perceptual grouping ability in Williams syndrome: Evidence for deviant patterns of performance. Neuropsychologia, 43, 815-822.

Farran, E.K., Jarrold, C., Gathercole, S.E. (2001). Block Design performance in the Williams Syndrome phenotype: A problem with mental imagery? Journal of Child Psychology and Psychiatry, 42(6), 719-728.

Farran, E.K., Jarrold, C., \& Gathercole, S.E. (2003). Divided attention, selective attention and drawing: processing preferences in Williams syndrome are dependent on the task administered. Neuropsychologia, 41, 676-687. 
Feeney, S.M., \& Stiles, J. (1996). Spatial analysis: An examination of preschoolers' perception and construction of geometric patterns. Developmental Psychology, 32(5), 933-941.

Ferrero, G.B., Biamino, E., Sorasio, L., Banaudi, E., Peruzzi, L., Forzano, S., di Cantogno, L.V., \& Silengo, M.C. (2007). Presenting phenotype and clinical evaluation in a cohort of 22 Williams-Beuren syndrome patients. European Journal of Medical Genetics, 50, 327-337.

Gentaz, E., Luyat, M., Cian, C., Hatwell, Y., Barraud, P.A., \& Raphel, C. (2001). The reproduction of vertical and oblique orientations in the visual, haptic, and somatovestibular systems. The Quarterly Journal of Experimental Psychology Section A, 54(2), 513 - 526.

Georgopoulos, M.-A., Georgopoulos, A.P., Kuz, N., \& Landau, B. (2004). Figure copying in Williams syndrome and normal subjects. Experimental Brain Research, 157, 137146.

Hoffman, J.E., Landau, B., \& Pagani, B. (2003). Spatial breakdown in spatial construction: Evidence from eye fixations in children with Williams syndrome. Cognitive Psychology, 46, 260-301.

Jarrold, C., Baddeley, A.D., \& Hewes, A.K. (1998). Verbal and nonverbal abilities in the Williams syndrome phenotype: Evidence for diverging developmental trajectories. Journal of Child Psychology and Psychiatry, 39(4), 511-523.

Karmiloff-Smith, A. (1990). Constraints on representational change: Evidence from children's drawing. Cognition, 34(1), 57-83.

Karmiloff-Smith, A., Brown, J.H., Grice, S., \& Paterson, S. . (2003). Dethroning the myth: Cognitive dissociations and innate modularity in Williams syndrome. Developmental Neuropsychology 23, 229-244. 
Levi, D.M. (2008). Crowding-An essential bottleneck for object recognition: A mini-review. Vision Research, 48(5), 635-654.

Magnan, A., Baldy, R., \& Chatillon, J.-F. (1999). Organizing principle in 4- to 8-year-old children's drawings of embedded geometric shapes. Swiss Journal of Psychology, 58(1), 3-11.

Menghini, D., Addona, F., Costanzo, F., \& Vicari, S. (2010). Executive functions in individuals with Williams syndrome. Journal of Intellectual Disability Research, $54(5), 418-432$.

Mervis, C.B., \& John, A.E. (2008). Vocabulary abilities of children with Williams syndrome: Strengths, weaknesses, and relation to visuospatial construction ability. Journal of Speech, Language, and Hearing Research, 51(4), 967-982.

Miller, P. H. (1990). The development of strategies of selective attention. In Bjorklund, D.F. (Ed.), Children's strategies: Contemporary views of cognitive development (pp. 157184). Hillsdale, NJ: Erlbaum.

Morris, C.A., Demsey, S.A., Leonad, C.O., Dilts, C., \& Blackburn, B.L. (1988). The natural history of Williams syndrome: Physical characteristics. Journal of Pediatrics, 113, $318-326$.

Nickerson, E., Greenberg, F., Keating, M.T., McCaskill, C., \& Shaffer, L.G. (1995). Deletions of the elastin gene at 7q11.23 occur in $~ 90 \%$ of patients with Williams syndrome. American Journal of Human Genetics, 56(5), 1156-1161.

Ninio, A., \& Lieblich, A. (1976). The grammar of action: "Phase structure" in children's copying. Child Development, 47(3), 846-849.

Palomares, M., Landau, B., \& Egeth, H. (2009). Orientation perception in Williams syndrome: Discrimination and integration. Brain and Cognition, 70(1), 21-30. 
Pani, J.R., Mervis, C.B., \& Robinson, B.F. (1999). Global spatial organization by individuals with Williams syndrome. Psychological Science, 10, 453-458.

Phillips, W.A., Hobbs, S.B., \& Pratt, F.R. (1978). Intellectual realism in children's drawings of cubes. Cognition, 6(1), 15-33.

Piaget, J. (1948). The Child's conception of space. London: Routledge and Kegan Paul Ltd.

Raven, J.C. (1993). Coloured progressive matrices. Oxford, UK: Information Press, Ltd.

Satlow, E., \& Newcombe, N. (1998). When is a triangle not a triangle? Young children's developing concepts of geometric shape. Cognitive Development, 13(4), 547-559.

Siegler, R.S. (1987). The perils of averaging data over strategies: An example from children's addition. Journal of Experimental Psychology: General, 116, 250-264.

Siegler, R.S., Adolph, K.E., \& Lemaire, P. (1996). Strategy choices across the lifespan. In L. Reder (Ed.), Implicit memory and metacognition (pp. 79-121). Mahwah, NJ: Erlbaum.

Smoot, L., Zhang, H., Klaiman, C., Schultz, R., \& Pober, B. (2005). Medical overview and genetics of Williams-Beuren syndrome. Progress in Pediatric Cardiology, 20, 195205.

de Souza, D.H., Moretti-Ferreira, D., \& Rugolo, L.M.S. de S. (2007). Fluorescence in situ hybridization (FISH) as a diagnostic tool for Williams-Beuren Syndrome. Genetics and Molecular Biology, 30, 17-20.

Stinton, C., Farran, E.K., \& Courbois, Y. (2008). Mental rotation in Williams syndrome: An impaired ability. Developmental Neuropsychology, 33(5), 565-583.

Strømme, P., Bjømstad, P.G., \& Ramstad, K. (2002). Prevalence estimation of Williams syndrome. Journal of Child Neurology, 17(4), 269-271.

Tada, W. L., \& Stiles, J. (1996). Developmental change in children's analysis of spatial patterns. Developmental Psychology, 32(5), 951-970. 
Tassabehji, M. (2003). Williams-Beuren syndrome: A challenge for genotype-phenotype correlations. Human Molecular Genetics, 12(2), 229-237.

Thomas, M. S. C., Grant, J., Barham, Z., Gsödl, M., Laing, E., Lakusta, L., Tyler, L. K., Grice, S., Paterson, S. \& Karmiloff-Smith, A. (2001). Past tense formation in Williams syndrome. Language and Cognitive Processes, 16(2/3), 143-176.

Toomela, A. (2002). Drawing as a verbally mediated activity: A study of relationships between verbal, motor, and visuospatial skills and drawing in children. International Journal of Behavioral Development, 26(3), 234-247.

Witkin, H.A., Oltman, P.K., Raskin, E., \& Karp, S.A (1971). A manual for the Embedded Figures Test. Palo Alto, CA: Consulting Psychologists Press, Inc.

Woody-Dorning, J., \& Miller, P.H. (2001). Children's individual differences in capacity: Effects on strategy production and utilization. British Journal of Developmental Psychology, 19, 543-557. 
Table 1

Participant Chronological Age, BPVS and RCPM Scores.

\begin{tabular}{|c|c|c|c|c|}
\hline & \multicolumn{2}{|c|}{ Williams Syndrome $(n=19)$} & \multicolumn{2}{|c|}{ Typically Developing $(n=19)$} \\
\hline & $\operatorname{Mean}(S D)$ & Range & $\operatorname{Mean}(S D)$ & Range \\
\hline CA (years; months) & $25 ; 3(11 ; 0)$ & $8 ; 9-42 ; 6$ & $6 ; 3(0 ; 6)$ & $5 ; 3-7 ; 6$ \\
\hline RCPM Score & $18.68(5.56)$ & $10-33$ & $19.37(5.41)$ & $14-30$ \\
\hline BPVS Score & $104.21(22.45)$ & $47-124$ & $73.84(13.03)$ & $52-99$ \\
\hline
\end{tabular}


Table 2.

Use of Each Strategy by Both Groups in Experiment One: Drawing and Constructing Intersecting Shapes

Drawing

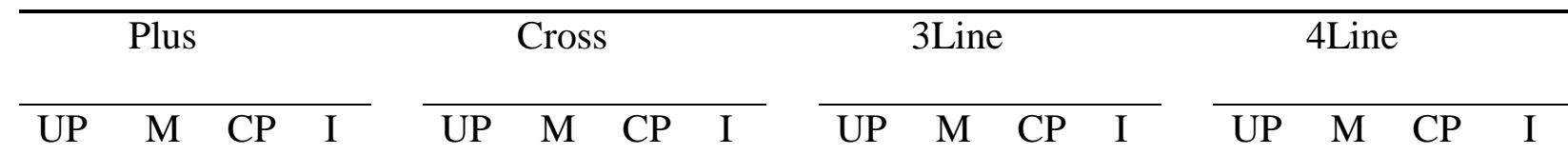

$\begin{array}{lllllllllllllllll}\text { WS } & 19 & 0 & 0 & 0 & 19 & 0 & 0 & 0 & 11 & 4 & 0 & 4 & 5 & 4 & 1 & 9 \\ \text { TD } & 19 & 0 & 0 & 0 & 18 & 1 & 0 & 0 & 13 & 5 & 1 & 0 & 14 & 3 & 2 & 0\end{array}$

Construction

\begin{tabular}{|c|c|c|c|c|c|c|c|c|c|c|c|c|c|c|c|c|}
\hline & \multicolumn{4}{|c|}{ Plus } & \multicolumn{4}{|c|}{ Cross } & \multicolumn{4}{|c|}{ 3Line } & \multicolumn{4}{|c|}{ 4Line } \\
\hline & UP & $\mathrm{M}$ & $\mathrm{CP}$ & I & UP & $\mathrm{M}$ & $\mathrm{CP}$ & I & UP & $\mathrm{M}$ & $\mathrm{CP}$ & I & UP & $\mathrm{M}$ & $\mathrm{CP}$ & I \\
\hline WS & 14 & 2 & 2 & 1 & 13 & 3 & 1 & 2 & 11 & 1 & 0 & 7 & 8 & 0 & 1 & 10 \\
\hline $\mathrm{TD}$ & 19 & 0 & 0 & 0 & 19 & 0 & 0 & 0 & 13 & 0 & 4 & 2 & 14 & 2 & 0 & 3 \\
\hline
\end{tabular}

UP- Unsegmented Part strategy, M- Mixed strategy, CP- Central Point strategy, I- Incomplete 
Figure Captions

Figure 1. Drawing and Constructing Simple Shapes task figures (junction control figures Lateral-T and Slanted-T).

Figure 2. Figures used in the Drawing and Constructing Complex Shapes task.

Figure 3. Percentage of participants' starting location when the CEP was not used: Mean (S.E.) 
Fig. 1.

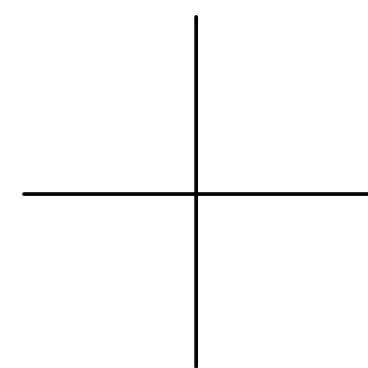

Plus

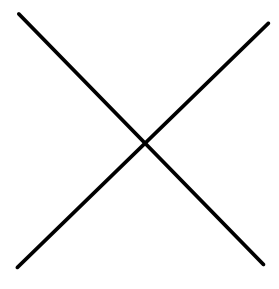

Cross

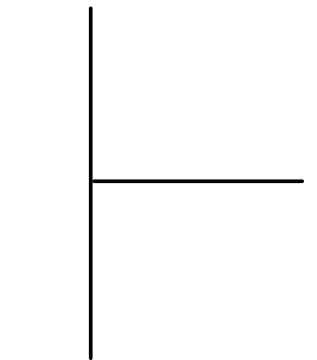

Lateral-T

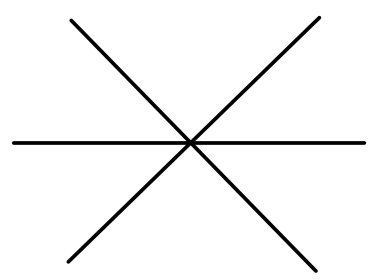

Three-Line

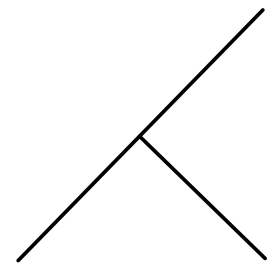

Slanted-T

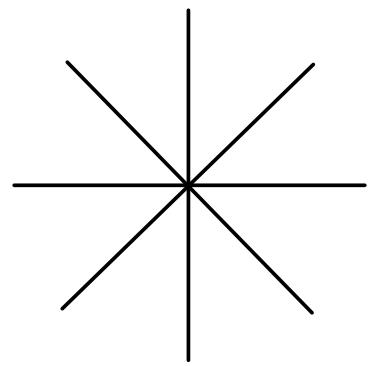

Four-Line 
Fig. 2.
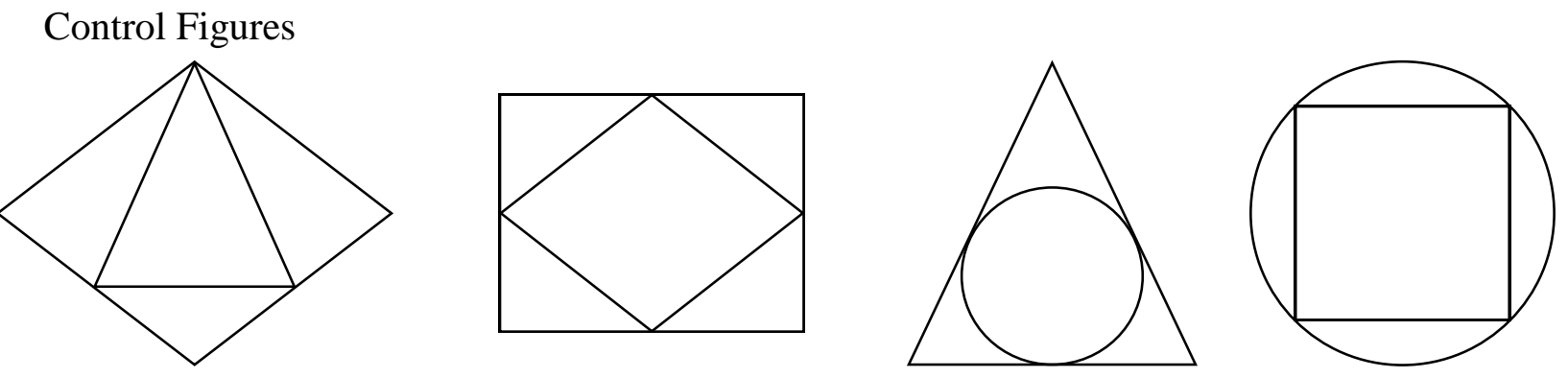

Experimental Figures
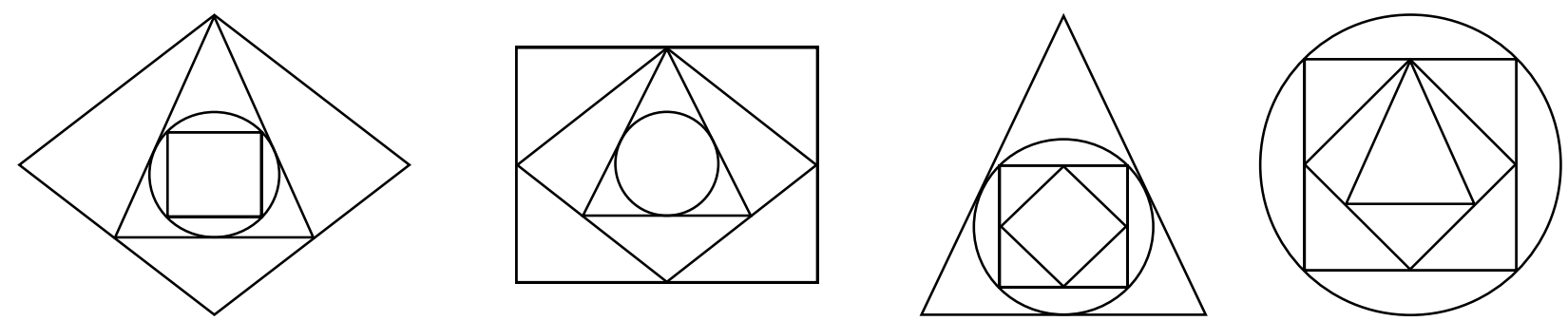
Fig. 3.

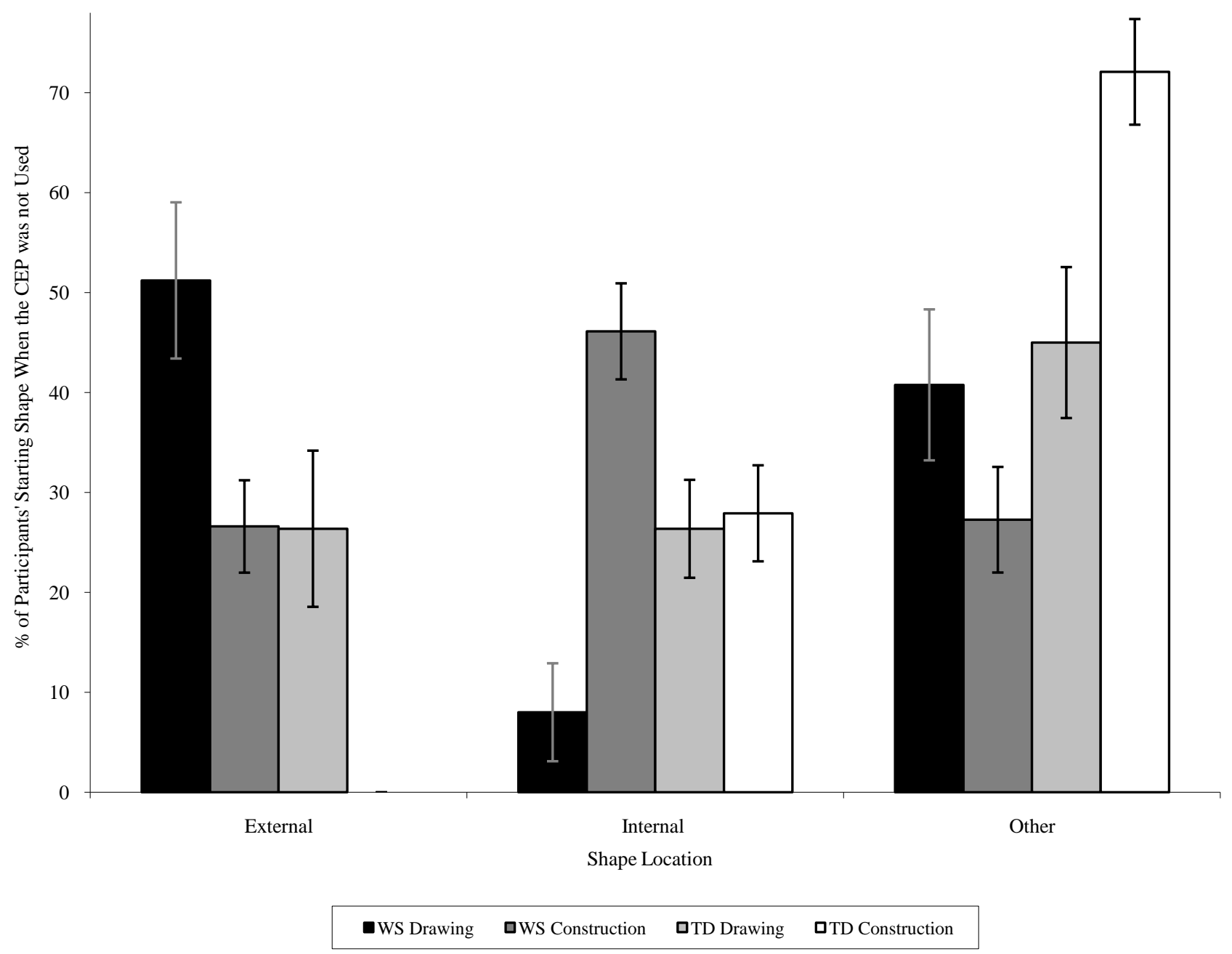

